\title{
miR-96 promotes tumor proliferation and invasion by targeting RECK in breast cancer
}

\author{
JUNFENG ZHANG ${ }^{1}$, XIANGJIE KONG ${ }^{1}$, JIA LI ${ }^{2}$, QIFENG LUO ${ }^{1}$, \\ XIAOYU LI ${ }^{1}$, LEI SHEN $^{3}$, LEI CHEN ${ }^{1}$ and LIN FANG ${ }^{1}$ \\ ${ }^{1}$ Department of General Surgery, Shanghai Tenth People's Hospital, Tongji University School of Medicine, \\ Shanghai 200072, P.R. China; ${ }^{2}$ Department of Microbiology and Genetic Institute, University of Paris 11, Paris, France; \\ ${ }^{3}$ Department of General Surgery, Shanghai East Hospital, Tongji University School of Medicine, Shanghai 200072, P.R. China
}

Received November 12, 2013; Accepted December 9, 2013

DOI: $10.3892 / o r .2013 .2934$

\begin{abstract}
RNAs (miRNAs) are small non-coding RNAs that post-transcriptionally regulate gene expression in diverse biological processes. The aim of the present study was to investigate the expression pattern of miR-96 in breast cancer and its biological role in tumor progression. The expression levels of miR-96 were analyzed in 38 breast cancer specimens and 6 breast cancer cell lines by quantitative reverse-transcription polymerase chain reaction (qRT-PCR). The effect of miR-96 on proliferation was evaluated by MTT assays, and cell migration and invasion were evaluated by Transwell assays in MDA-MB231 human breast cancer cells. Luciferase reporter assays were performed to validate the regulation of a putative target of miR-96. The effects of modulating miR-96 on endogenous levels of this potential target were subsequently confirmed via qRT-PCR and western blot analysis. We found that expression of miR-96 was commonly upregulated in breast cancer cells and breast cancer specimens when compared with that in nonmalignant breast epithelial cells and adjacent normal tissues. Ectopic expression of miR-96 promoted cellular proliferation, migration and invasion of breast cancer cells, whereas inhibition of miR-96 suppressed those functions. Luciferase assays revealed that miR-96 directly bound to the 3'-untranslated region (3'-UTR) of RECK. qRT-PCR and western blot analysis confirmed that miR-96 regulated the expression of RECK both at the mRNA and protein levels. Knockdown of RECK expression in MDA-MB-231 cells by siRNA significantly promoted cell proliferation, migration and invasion. Collectively, miR-96 was significantly upregulated in breast cancer. Our data also delineate the molecular pathway by which miR-96 promotes breast cancer proliferation, migration and invasion. Our findings may have important implications for the treatment of breast cancer.
\end{abstract}

Correspondence to: Professor Lin Fang, Department of General Surgery, Shanghai Tenth People's Hospital, Tongji University School of Medicine, Shanghai 200072, P.R. China

E-mail: zjfdipan@126.com

Key words: miR-96, RECK, proliferation, invasion, breast cancer

\section{Introduction}

microRNAs (miRNAs) are small, endogenous, non-coding RNA molecules (19-23 nucleotides) that regulate gene expression post-transcriptionally by negatively regulating the stability or translational efficiency of their target mRNAs (1). Aberrant miRNA expression plays diverse roles in tumorigenesis and tumor development through modulation of cell proliferation, differentiation, apoptosis, motility and malignant transformation (2). Additionally, deregulated miRNAs exhibit oncogenic or tumor suppressor properties (3). For example, among other findings, miR-24 and miR-21 have been shown to influence breast cancer invasion and metastasis $(4,5)$. Overexpression of miR-187 has been associated with lymph node metastasis and poor prognosis in breast cancer (6). miR-96 was found to be highly upregulated in a variety of tumors, including prostate cancer (7), hepatocellular carcinoma (8), lung (9), colorectal (10), endometrial (11) and breast cancer (12). Furthermore, overexpression of miR-96 induced cell proliferation in MCF-7 breast cancer cells by targeting FOXO3a (12). However, to date, the biological function of miR-96 in breast cancer carcinogenesis remains largely unknown.

RECK (reversion-inducing cysteine-rich protein with Kazal motifs), a ubiquitous tumor-suppressor gene, negatively regulates MMP-9, MMP-2 and MT1-MMP, and has a significant effect on the regulation of angiogenesis, tumor invasion and metastasis $(13,14)$. The functional inactivation of RECK by regulation of its expression has been observed in various types of solid tumors, including breast cancer (15). Furthermore, RECK was recently described as a potentially useful prognostic marker for breast cancer (16). Therefore, overexpression of RECK should be considered as a therapeutic approach for breast cancer. Recently, several miRNAs, miR-92a (17), miR-182 (18), miR-15a (19) and miR-21 (20) were reported to suppress RECK expression and function as oncogenes. In addition, miR-222 has been shown to directly silence RECK and promote proliferation in $H$. pylori-associated gastric cancer (21). These data highlight the importance of miRNAs targeting RECK in breast cancer development and provide insight into the mechanisms underlying tumorigenesis.

In the present study, we reported that miR-96 was significantly upregulated in breast cancer cells and breast cancer 
specimens when compared with that in non-malignant breast epithelial cells and adjacent normal tissues. Ectopic expression of miR-96 promoted cellular proliferation, migration and invasion of MDA-MB-231 cells, at least in part, by targeting RECK. Moreover, we found that the silencing of RECK by RNA interference mimicked the oncogenic effects of miR-96. Collectively, the present study indicates that miR-96 serves as an oncogene in breast cancer and is a vital regulator of cellular proliferation, migration and invasion. Targeting miR-96 is a potential novel strategy for the treatment of human breast cancer.

\section{Materials and methods}

Specimens. In the present study, 38 paired breast cancer specimens and adjacent normal breast tissues were collected from the Department of General Surgery of the Shanghai Tenth People's Hospital. These samples were immediately snapfrozen in liquid nitrogen. Both tumor and normal tissues were histologically confirmed by two different experienced pathologists according to the World Health Organization (WHO) using H\&E (hematoxylin and eosin) staining. No patients received chemotherapy or radiotherapy prior to surgery.

Cell lines and transfection. The human breast cancer cell lines MDA-MB-231, MCF-7, MDA-MB-468, MDA-MB-435, T-74D, MDA-MB-453 and non-malignant breast epithelial cell line MCF-10A were all obtained from the American Type Culture Collection (ATCC; Manassas, VA, USA). The breast cancer cells were cultured in Dulbecco's modified Eagle's medium (DMEM) (Gibco, Carlsbad, CA, USA) supplemented with $10 \%$ fetal bovine serum (FBS) (Gibco), penicillin (100 U/ml) and streptomycin $(100 \mu \mathrm{g} / \mathrm{ml})$ (Enpromise, China). MCF-10A cells were cultured in Mammary Epithelial Basal Medium (MEBM) (Cambrex). Cells were incubated at $37^{\circ} \mathrm{C}$ in a humidified chamber supplemented with $5 \% \mathrm{CO}_{2}$.

miR-96 mimics, inhibitors and their negative control (NC) were chemosynthesized by Shanghai Genepharma Co., Ltd. (Shanghai, China). The MDA-MB-231 cells were cultured to $\sim 30-40 \%$ confluence in 6-well plates and were transfected with miR-96 mimics or miR-96 inhibitors or RECK siRNA (Santa Cruz Biotechnology) at working concentrations using Lipofectamine 2000 (Invitrogen, Carlsbad, CA, USA), in accordance with the manufacturer's instructions. miR- and siRNA-negative control (NC) were used as negative controls. After $48 \mathrm{~h}$ of incubation, cells were harvested for further analysis. All transfections were performed in triplicates.

Quantitative reverse-transcription polymerase chain reaction ( $q R T-P C R)$. For detection of miR-96 expression, primer design and qRT-PCR were carried out according to a previously described method (22). miRNA was isolated from tissues and cells using the miRcute miRNA Isolation kit according to the manufacturer's instructions (Beijing Tianjin, Beijing, China). The primers for miR-96 were stem-loop RT primer 5'-GTCGTATCCAGTGCAGGGTCCGAGGTATTCGCACT GGATACGACAGCAAA-3'; forward 5'-GCCCGCTTTG GCACTAGCACATT-3' and reverse 5'-GTGCAGGGTCCGA GGT-3'. The primers for U6 small nuclear RNA were RT primer 5'-GTCGTATCCAGTGCAGGGTCCGAGGTGCAC
TGGATACGACAAAATATGG-3'; forward 5'-TGCGGGT GCTCGCTTCGGCAGC-3' and reverse 5'-CCAGTGCAGG GTCCGAGGT-3'. cDNA was generated by reverse transcription using the PrimeScript ${ }^{\mathrm{TM}}$ RT-PCR kit in accordance with the manufacturer's instructions (Takara, Tokyo, Japan). Realtime PCR was performed on a 7900HT Fast RT-PCR instrument (Applied Biosystems, Singapore). The amplification procedure was as follows: $5 \mathrm{~min}$ at $95^{\circ} \mathrm{C}$, followed by 40 cycles at $95^{\circ} \mathrm{C}$ for $30 \mathrm{sec}$ and $65^{\circ} \mathrm{C}$ for $45 \mathrm{sec}$.

For detection of RECK mRNA expression, total RNA was isolated using TRIzol (Invitrogen), and cDNA was generated by reverse transcription using the PrimeScript RT-PCR kit in accordance with the manufacturer's instructions (Takara). Real-time PCR was performed on a 7900HT Fast RT-PCR instrument using SYBR-Green and the following primers: RECK, 5'-AACCAAATGTGCCGTGAT-3' (sense), 5'-ATGG CTTGACAGTATTCTCG-3' (antisense); $\beta$-actin, 5'-CAGAG CCTCGCCTTTGCC-3' (sense), 5'-GTCGCCCACATAGGA ATC-3' (antisense). The PCR parameters for relative quantification were as follows: $2 \mathrm{~min}$ at $95^{\circ} \mathrm{C}$, followed by 40 cycles of $45 \mathrm{sec}$ at $57^{\circ} \mathrm{C}$ and $45 \mathrm{sec}$ at $72^{\circ} \mathrm{C}$. The relative expression was evaluated following the relative quantification equation, $2^{-\Delta \Delta \mathrm{CT}}$ (23). Each sample was tested in triplicate.

Cell proliferation assay. Cell proliferation was determined using an MTT assay kit (Sigma, Santa Clara, CA, USA) in accordance with the manufacturer's instructions. In brief, the transfected cells $\left(5 \times 10^{3}\right.$ cells/well) were seeded into 96-well culture plates (BD Biosciences, Franklin Lakes, NJ, USA) and incubated overnight at $37^{\circ} \mathrm{C}$ in $5 \% \mathrm{CO}_{2}$. Cell proliferation was assessed at 24, 48, 72 and $96 \mathrm{~h}$ following addition of $0.5 \mathrm{mg} /$ ml MTT (Sigma) solution. After a 4-h incubation, the medium was replaced with $100 \mu$ l dimethylsulfoxide (DMSO; Sigma) and vortexed for $10 \mathrm{~min}$. The optical density (OD) of each well was measured using a microplate reader at $490 \mathrm{~nm}$. Each experiment was performed in triplicate.

Cell migration and invasion assays. For the invasion assay, the miR-96 mimic- or miR-96 inhibitor (100 nmol/l)-transfected MDA-MB-231 cells $\left(4 \times 10^{4}\right.$ cells/Transwell) were plated in the top chamber of Transwells (Millipore) with a Matrigel (2 $\mathrm{mg} / \mathrm{ml}$ )-coated membrane containing 8-mm diameter pores in $200 \mu \mathrm{l}$ serum-free DMEM in triplicate. Medium containing $10 \%$ FBS was added to the lower chamber. After $48 \mathrm{~h}$ of incubation, cells remaining on the upper membrane surface were removed by cotton swab scrubbing; cells on the lower surface of the membrane were fixed in $10 \%$ formalin at room temperature for $30 \mathrm{~min}$ and stained with $0.5 \%$ crystal violet. The cell number in five random fields (x200) was counted for each chamber. The stained cells were dissolved in glacial acetic acid, and solutions were transferred to a 96-well culture plate for colorimetric reading of OD at $560 \mathrm{~nm}$. The OD value represents the invasive ability. For the migration assays, the infected cells ( $1 \times 10^{4}$ cells/Transwell) were plated in the top chamber with no Matrigel. After a 24-h incubation, the number of migrated cells was counted as described above. Each experiment was carried out in triplicate.

Luciferase assay. The 3'-UTR segments of the RECK mRNA sequence containing the predicted miR-96 binding sites were 
amplified by PCR in a total volume of $50 \mu 1$ using the PrimerStar kit (Takara) in accordance with the manufacturer's instructions. The primers used were 5'-AAACTAGCGGCC GCTAGTGCTGCTACTTATATAATTGCCAAAT-3' (sense) and 5'-CTAGATTTGGCAATTATATAAGTAGCAGCA CTAGCGGCCGCTAGTTT-3' (antisense). The mutant constructs were generated by mutation. Fragments were subcloned into the XhoI site in the 3'-UTR of Renilla luciferase of the psiCHECK-2 reporter vector. MDA-MB-231 cells were transiently cotransfected in 24-well plates with $0.2 \mu \mathrm{g}$ psiCHECK-2/RECK 3'-UTR or psiCHECK-2/RECK 3'-UTR mutant reporter plasmids and $100 \mathrm{nmol} / \mathrm{l} \mathrm{miR}-96$ or miR-NC using Lipofectamine ${ }^{\mathrm{TM}} 2000$ (Invitrogen). After 48 h, firefly and Renilla luciferase activities were measured by using a Dual Luciferase Assay (Promega, Madison, WI, USA). The firefly luciferase activity of each sample was normalized to the Renilla luciferase activity.

Western blot analysis. The protein expression levels were analyzed by western blot analysis. Cells were lysed in lysis buffer (10 mmol/1 Tris-HCl, pH 7.4, 1\% NP-40, 0.1\% deoxycholic acid, $0.1 \%$ SDS, $150 \mathrm{mmol} / 1 \mathrm{NaCl}, 1 \mathrm{mM}$ EDTA and $1 \%$ protease inhibitor cocktail) (Sigma). The protein concentrations were quantified using a BCA protein assay kit (Pierce). Protein was separated using $8 \%$ sodium dodecyl sulfate polyacrylamide gel electrophoresis and transferred to a nitrocellulose membrane (Beyotime Institute of Biotechnology, Jiangsu, China). The membrane was immunoblotted overnight at $4^{\circ} \mathrm{C}$ with primary antibodies against RECK $(1: 1,000$ dilution; no. 3433, Cell Signaling Technology) and $\beta$-actin (1:1,000 dilution; sc-1616-R, Santa Cruz Biotechnology), as a loading control. Horseradish peroxidase-conjugated secondary antibodies was incubated with the membrane for $2 \mathrm{~h}$ at $37^{\circ} \mathrm{C}$ after three washes with TBST. Immunoreactive protein bands were detected with an Odyssey Scanning system.

Statistical analysis. Data are presented as the means \pm standard deviation (SD) from at least three independent experiments. The t-test (two-tailed) was used to draw a comparison between groups. P-values $<0.05$ were considered to indicate statistically significant results.

\section{Results}

miR-96 is upregulated in human breast cancer cell lines and clinical specimens. We examined the miR-96 expression in breast cancer cell lines MDA-MB-231, MCF-7, MDA-MB435, T-74D, MDA-MB-468 and MDA-MB-453 as well as breast cancer tissues. As shown in Fig. 1A, all breast cancer cell lines expressed higher levels of miR-96 when compared with the levels in the non-malignant breast epithelial cell line MCF-10A. Furthermore, we compared miR-96 expression profiles between 38 pairs of breast cancer tissues and matched adjacent normal breast tissues. In comparison with the adjacent normal breast tissues, miR-96 showed on average a 3.8-fold higher expression in cancer tissues $(\mathrm{P}<0.05$; Fig. 1B). These results indicated that miR-96 is upregulated in breast cancer.

miR-96 promotes MDA-MB-231 cell proliferation. To investigate the effects of miR-96 on breast cancer cell proliferation.
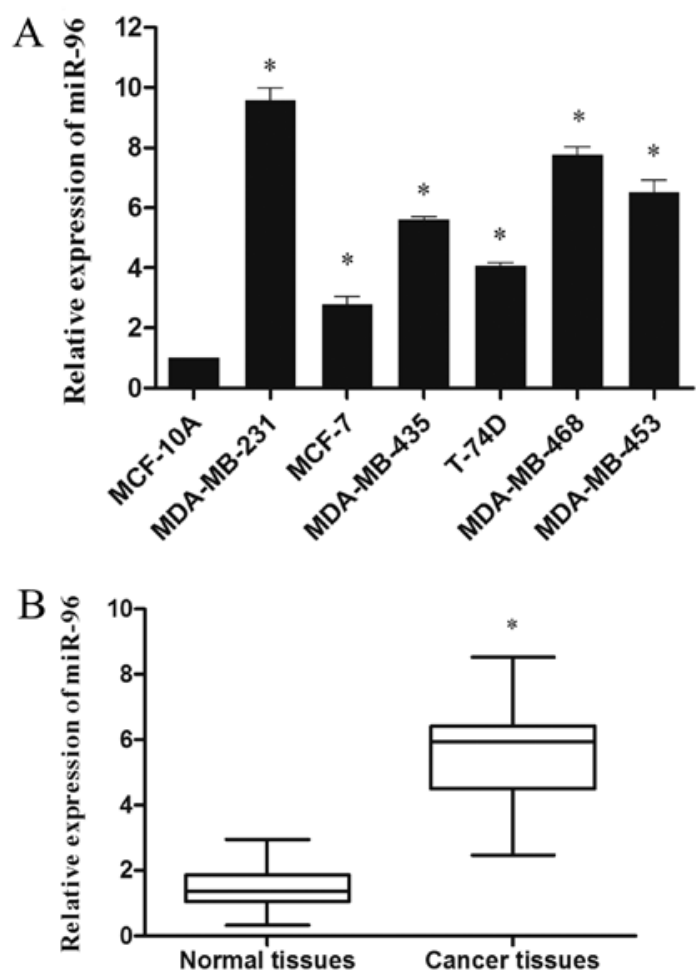

Figure 1. miR-96 is upregulated in human breast cancer cell lines and clinical specimens. The levels of miR-96 were measured by qRT-PCR and normalized to the expression of U6. (A) Relative expression of miR-96 in breast cancer cell lines MDA-MB-231, MCF-7, MDA-MB-435, T-74D, MDA-MB-468 and MDA-MB-453 compared to MCF-10A. (B) Relative miR-96 expression in 38 paired breast cancer tissues (Cancer tissues) and matched adjacent normal breast tissues (Normal tissues). Data represent the $2^{-\Delta \Delta C t}$ values $\pm \mathrm{SD} ;{ }^{*} \mathrm{P}<0.05$.

Initially, we assessed miR-96 expression in MDA-MB-231 cells transfected with miR-96 mimics or inhibitors $(100 \mathrm{nmol} / 1$ for 48 h) by qRT-PCR (Fig. 2A). As shown in Fig. 2B, upregulation of miR-96 significantly increased the growth rate of MDA-MB-231 cells at $48 \mathrm{~h}(18 \%), 72 \mathrm{~h}(26 \%)$ and $96 \mathrm{~h}(13 \%)$ compared with the negative control $(\mathrm{P}<0.05)$. Moreover, MTT assay showed that suppression of miR-96 markedly inhibited the growth rate of MDA-MB-231 cells at $48 \mathrm{~h}(11 \%), 72 \mathrm{~h}(14 \%)$ and $96 \mathrm{~h}(12 \%)$ as compared with the control cells $(\mathrm{P}<0.05)$. The dose-dependent effect of miR-96 mimics was also evident in the MTT assay at $72 \mathrm{~h}$ (Fig. 2C). Taken together, these results revealed that miR-96 promotes the proliferation of MDA-MB-231 breast cancer cells.

miR-96 promotes MDA-MB-231 cell migration and invasion. To investigate whether miR-96 affects the migratory and invasive abilities of the cells, we transfected MDA-MB-231 cells with $100 \mathrm{nmol} / 1 \mathrm{miR}-96$ mimics or inhibitors and detected changes in their motility using Transwell assays. Transwell migration assays performed in the absence of Matrigel showed that overexpression of miR-96 promoted MDA-MB-231 cell migration by 1.5 -fold compared to the control cells. In contrast, MDA-MB-231 cell migratory activity was significantly inhibited when cells were transfected with the miR-96 inhibitors compared to the control $(0.58$-fold; $\mathrm{P}<0.05)$ (Fig. 3A and $\mathrm{B})$. The upregulation of miR-96 significantly promoted the invasion of MDA-MB-231 cells as measured by Transwell invasion assays that included $2 \mathrm{mg} / \mathrm{ml}$ Matrigel (1.7-fold; P<0.05). In 

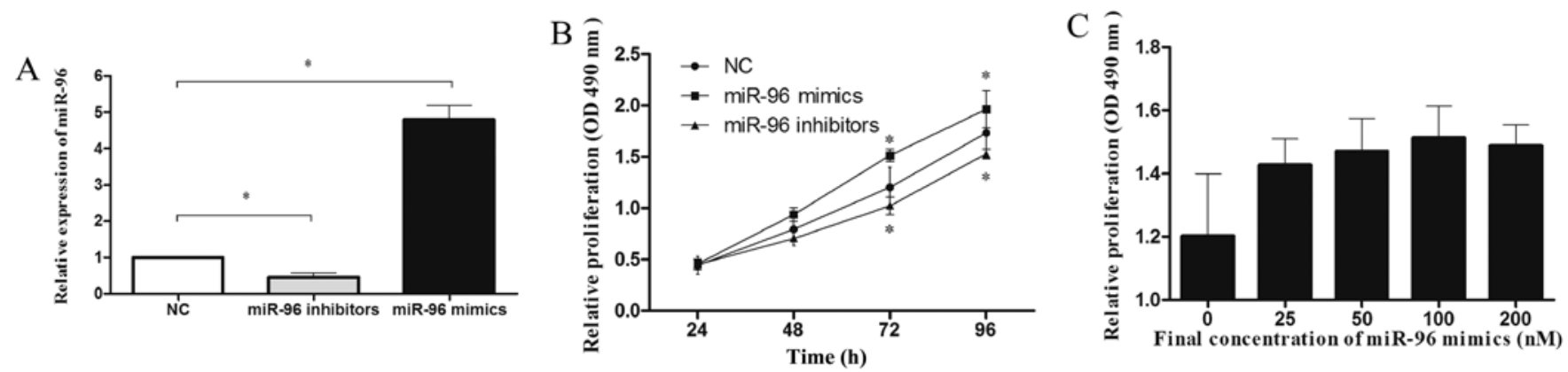

Figure 2. miR-96 promotes MDA-MB-231 cell proliferation. (A) MDA-MB-231 cells were transfected with miR-96 mimics or inhibitors or miR-NC at $100 \mathrm{nmol} / 1$ for $48 \mathrm{~h}$. Relative miR-96 expression was measured by qRT-PCR. (B) MDA-MB-231 cells were transfected with miRNAs at $100 \mathrm{nmol} / 1$. The MTT assay was performed to monitor the proliferation level of MDA-MB-231 cells at 24, 48, 72 and $96 \mathrm{~h}$. (C) Cells transfected with the indicated concentrations of miR-96 mimics were subjected to MTT assay at $72 \mathrm{~h}$. The optical density of each well was measured at the indicated time-points with a microplate reader at $490 \mathrm{~nm}$. Graph represents OD $490 \mathrm{~nm} \pm \mathrm{SD} ;{ }^{*} \mathrm{P}<0.05$.
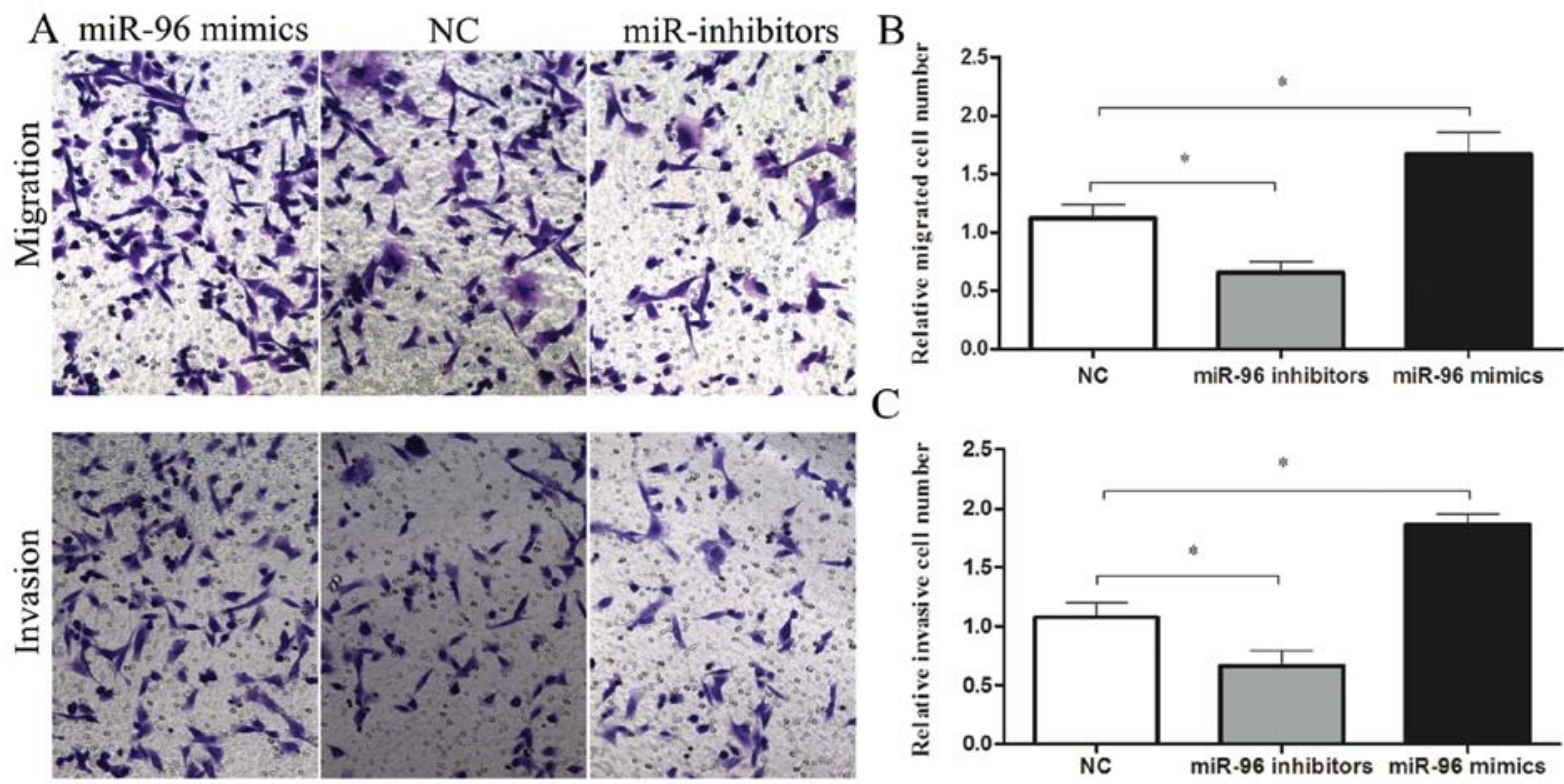

Figure 3. miR-96 promotes MDA-MB-231 cell migration and invasion. We transfected MDA-MB-231 cells with $100 \mathrm{nmol} / 1 \mathrm{miR}-96 \mathrm{mimics}$ or inhibitors and detected changes in their motility using Transwell assays. (A) Representative images of crystal violet-stained migratory or invasive MDA-MB-231 cells on the membrane. (B) Quantification of the migrated cells by solubilization of crystal violet and spectrophotometric reading at OD 573. (C) Quantification of the invasive cells. Data represent mean $\pm \mathrm{SD},{ }^{*} \mathrm{P}<0.05$.

addition, a decrease in the invasive ability of miR-96 inhibitortransfected cells when compared with the control (0.61-fold; $\mathrm{P}<0.05)$ indicated that miR-96 also participated in human breast cancer cell invasion (Fig. 3A and C).

miR-96 downregulates RECK expression by binding the 3'UTR of RECK. To explore the mechanism by which miR-96 functions in breast cancer, we searched for putative targets using the TargetScan database. We identified a binding site for miR-96 in the 3'-UTR of RECK mRNA. To validate whether RECK is a bona fide target of miR-96, we cloned the 3'-UTR of RECK containing the putative miR-96 binding site into a luciferase reporter construct, in addition to a mutated RECK 3'-UTR (Fig. 4A). In comparison with the negative control, miR-96 mimics (100 nmol/l) significantly decreased the relative luciferase activity when co-transfected with the psiCHECK-2/RECK 3'-UTR. However, this effect of miR-96 was abolished following co-transfection of psiCHECK-2/RECK 3'-UTR mutant and miR-96 (Fig. 4B).

To determine whether miR-96 regulates RECK at both the mRNA and protein levels, miR-96 inhibitors or mimics $(100 \mathrm{nmol} / \mathrm{l})$ were transfected into MDA-MB-231 cells, and the levels of RECK mRNA and protein were monitored $(\mathrm{P}<0.05$; Fig. 4C and D). qRT-PCR analysis revealed that inhibition of miR-96 in MDA-MB-231 cells led to increased expression of endogenous RECK mRNA when compared with the control. Additionally, western blot analysis showed that RECK protein expression was clearly upregulated following transfection of MDA-MB-231 cells with miR-96 inhibitors. In addition, enforced expression of miR-96 in MDA-MB-231 cells triggered a significant silencing effect on endogenous RECK expression, both at the mRNA and protein levels. Together, these data revealed that RECK is a novel target of miR-96. 
A

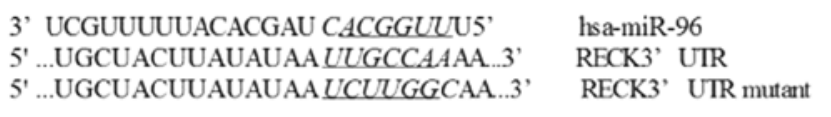

B

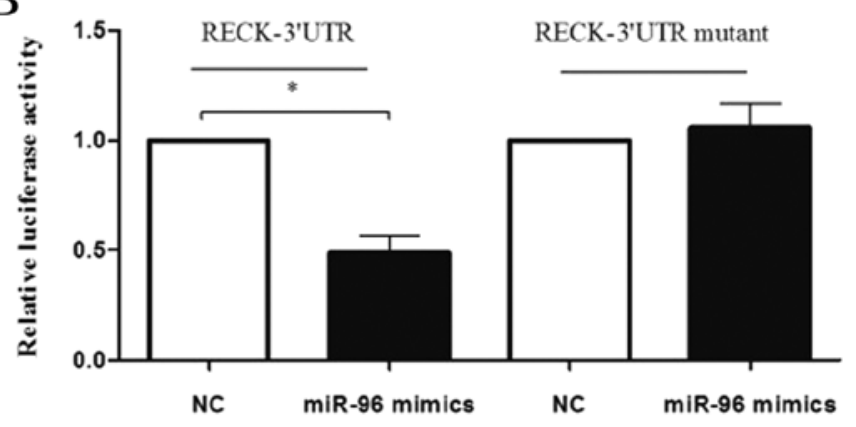

C

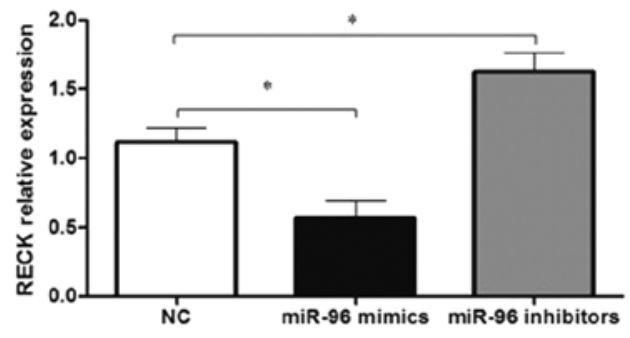

$\mathrm{D}$

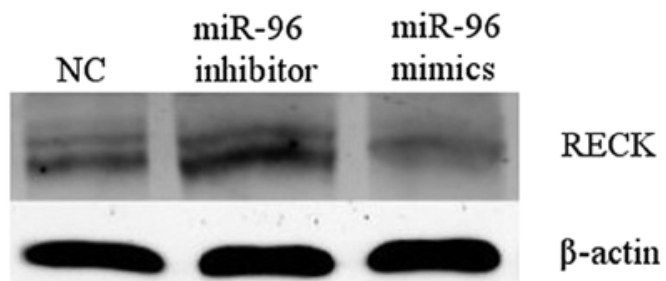

Figure 4. miR-96 downregulates RECK expression by binding the 3'-UTR of RECK. (A) The binding site for miR-96 in the 3'-UTR of RECK mRNA. (B) The relative luciferase activity (Renilla/firefly) was measured in MDA-MB-231 cells after co-transfection of the RECK 3'-UTR or RECK 3'-UTR mutant luciferase construct with either miR-96 mimics or miR-NC. (C) qRT-PCR analysis was used to detect RECK mRNA expression levels. (D) Western blot analysis was used to detect RECK protein expression levels. Data represent means $\pm \mathrm{SD} ;{ }^{*} \mathrm{P}<0.05$.

A

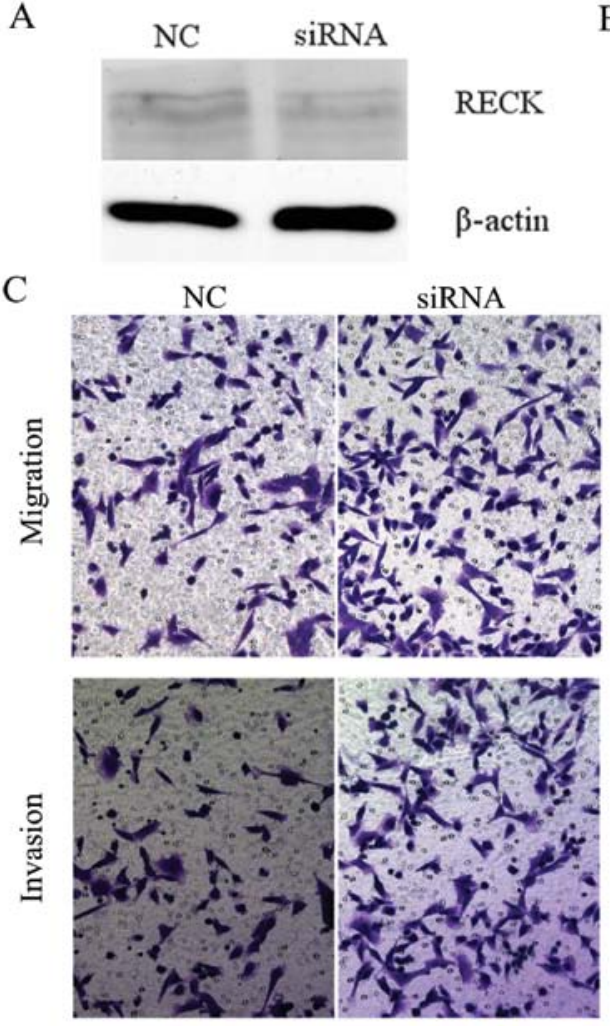

B

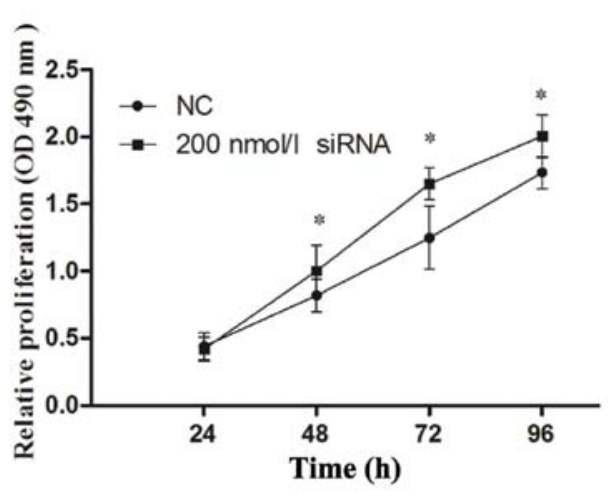

$\mathrm{D}$

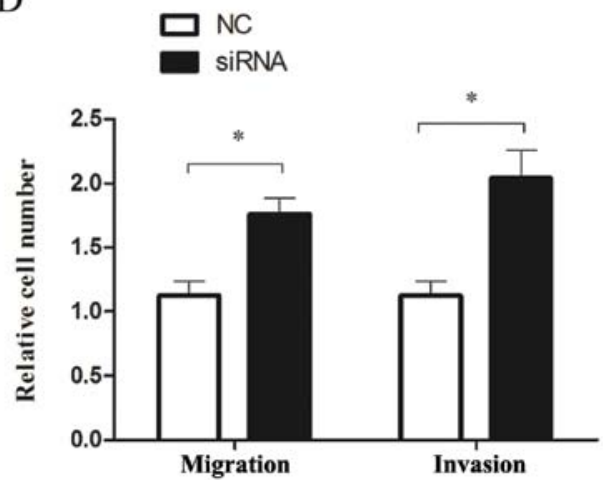

Figure 5. Effects of RECK on breast cancer cell proliferation and invasion. (A) Western blot analysis was used to detect RECK protein levels in MDA-MB-231 cells after transfection with $200 \mathrm{nmol} / \mathrm{l}$ RECK siRNA or siRNA-NC. (B) The MTT assay was performed to monitor the proliferation level of MDA-MB-231 cells at 24, 48,72 and $96 \mathrm{~h}$. (C) Representative images of crystal violet-stained migratory or invasive MDA-MB-231 cells on the membrane. (D) Quantification of the migratory or invasive cells by solubilization of crystal violet and spectrophotometric reading at OD 573. Data represent means \pm SD; ${ }^{*} \mathrm{P}<0.05$.

Effects of RECK on breast cancer cell proliferation and invasion. Since overexpression of miR-96 promoted the proliferation and invasion of MDA-MB-231 breast cancer cells, and given that RECK is a direct target of miR-96, we hypothesized that RECK is involved in the miR-96-promotion of proliferation and invasion by miR-96. The results indicated that protein level of RECK was decreased in the MDA-MB-231 cells transfected with $200 \mathrm{nmol} / 1$ RECK siRNA (Fig. 5A). 
Furthermore, RECK siRNA transfectants markedly increased cell proliferation by 22,32 and $16 \%$ at 48,72 and $96 \mathrm{~h}$, respectively, compared with the control siRNA ( $\mathrm{P}<0.05$; Fig. 5B). In addition, inhibition of RECK promoted the ability of cells to migrate and invade adjacent tissues $(\mathrm{P}<0.05$; Fig. $5 \mathrm{C}$ and $\mathrm{D})$. These data indicate that downregulation of RECK expression promotes cell proliferation, migration and invasion, phenocopying the overexpression of miR-96 in MDA-MB-231 cells.

\section{Discussion}

Growing evidence indicates that miRNAs may contribute to cancer pathogenesis. Dysregulation of miRNAs is associated with the initiation and progression of breast cancer, since they may serve as oncogenes or tumor suppressors (24-26). In the present study, we performed qRT-PCR to evaluate the expression pattern of miR-96 in breast cancer tissues and cell lines. We also investigated the biological impact of miR-96 and explored the molecular mechanisms by which miR-96 modulates the behavior of breast cancer cells.

Our results showed that miR-96 was dramatically upregulated in breast cancer tissues when compared to the expression in adjacent normal tissues. In addition, we demonstrated that miR-96 expression was elevated in 6 breast cancer cell lines when compared with the MCF-10A non-malignant breast epithelial cell line. Similar findings were reported in many different solid tumors, including hepatocellular carcinoma (8), lung (9), colorectal (10), endometrial (11), as well as in prostate cancer cell lines (7). These data indicate that the dysregulation of miR-96 might be a common occurrence in human cancer tissues and cell lines. In contrast, two studies reported the opposite expression pattern of miR-96 in pancreatic cancer, T-cell lymphoma and lung cancer $(27,28)$. Therefore, the expression profiles of miR-96 may be tissue- and cell typespecific. Further research is warranted to investigate the functional role and detailed mechanism of miR-96 in breast cancer.

To assess the role of miR-96 in breast cancer, we investigated the gain-or-loss of function effects of miR-96 on various aspects of breast cancer biology. The present study demonstrated that targeted knockdown of miR-96 expression by miR-96 inhibitors in MDA-MB-231 cells led to significant inhibition of cellular proliferation, migration and invasion. Conversely, transfection of miR-96 mimics into MDA-MB-231 cells induced corresponding malignant tumor cell behaviors. Previous studies demonstrated that suppression of miR-96 expression might also inhibit clonogenicity and invasion in hepatocellular carcinoma cells $(8,29)$. Taken together, these results indicate that dysregulated expression of miR-96 may function as an oncogene in multiple cancers.

Identification of putative miRNA targets is critical for understanding their pleiotropic functions in tumorigenesis (30). In endometrial cancer, overexpression of miR-96 induced impaired apoptotic responses by directly repressing FOXO1 expression (11). A recent study demonstrated that miR-96 promoted cell proliferation via targeting FOXO3a, $227^{\text {Kipl }}$ and $\mathrm{p} 21^{\mathrm{Cip} 1}$ in breast cancer (12). In the present study, we identified RECK as a direct target of miR-96 in MDA-MB-231 cells. Furthermore, endogenous RECK expression, both at the mRNA and protein levels, was decreased in MDA-MB-231 cells trans- fected with miR-96 mimics, but increased in MDA-MB-231 cells transfected with miR-96 inhibitors. Together, these data indicate that miR-96 directly interacts with RECK mRNA and suppresses RECK protein expression. Additionally, silencing of RECK expression by siRNA in MDA-MB-231 cells significantly promoted cellular proliferation, migration and invasion, consistent with the results of ectopic miR-96 expression in the same cells. These findings support the hypothesis that RECK is a new target of miR-96.

In summary, our findings demonstrate that miR-96 is upregulated in breast cancer tissues and cell lines, and is able to promote cellular proliferation, migration and invasion via direct regulation of the expression of RECK, implying that miR-96 can serve as a potential therapeutic target for breast cancer.

\section{Acknowledgements}

This research was made possible with financial support from the National Natural Sciences Foundation of China, for the project 81272240 , and the Shanghai Science Committee Foundation (to L.F.) (no. STCSM 10411964700).

\section{References}

1. O'Hara SP, Mott JL, Splinter PL, Gores GJ and LaRusso NF: MicroRNAs: key modulators of post-transcriptional gene expression. Gastroenterology 136: 17-25, 2009.

2. Bartel DP: MicroRNAs: genomics, biogenesis, mechanism, and function. Cell 116: 281-297, 2004.

3. Bartel DP: MicroRNAs: target recognition and regulatory functions. Cell 136: 215-233, 2009.

4. Du WW, Fang L, Li M, et al: MicroRNA miR-24 enhances tumor invasion and metastasis by targeting PTPN9 and PTPRF to promote EGF signaling. J Cell Sci 126: 1440-1453, 2013.

5. Zhu S, Wu H, Wu F, Nie D, Sheng S and Mo YY: MicroRNA-21 targets tumor suppressor genes in invasion and metastasis. Cell Res 18: 350-359, 2008.

6. Mulrane L, Madden SF, Brennan DJ, et al: miR-187 is an independent prognostic factor in breast cancer and confers increased invasive potential in vitro. Clin Cancer Res 18: 6702-6713, 2012.

7. Haflidadottir BS, Larne O, Martin M, Persson M, Edsjo A, Bjartell A and Ceder Y: Upregulation of miR-96 enhances cellular proliferation of prostate cancer cells through FOXO1. PLoS One 8: e72400, 2013.

8. Xu D, He X, Chang Y, Xu C, Jiang X, Sun S and Lin J: Inhibition of miR-96 expression reduces cell proliferation and clonogenicity of HepG2 hepatoma cells. Oncol Rep 29: 653-661, 2013.

9. Zhu W, Liu X, He J, Chen D, Hunag Y and Zhang YK: Overexpression of members of the microRNA-183 family is a risk factor for lung cancer: a case control study. BMC Cancer 11: 393, 2011.

10. Bandres E, Cubedo E, Agirre X, et al: Identification by real-time PCR of 13 mature microRNAs differentially expressed in colorectal cancer and non-tumoral tissues. Mol Cancer 5: 29, 2006.

11. Myatt SS, Wang J, Monteiro LJ, et al: Definition of microRNAs that repress expression of the tumor suppressor gene FOXO1 in endometrial cancer. Cancer Res 70: 367-377, 2010.

12. Lin H, Dai T, Xiong H, et al: Unregulated miR-96 induces cell proliferation in human breast cancer by downregulating transcriptional factor FOXO3a. PLoS One 5: e15797, 2010.

13. Takahashi C, Sheng Z, Horan TP, et al: Regulation of matrix metalloproteinase- 9 and inhibition of tumor invasion by the membrane-anchored glycoprotein RECK. Proc Natl Acad Sci USA 95: 13221-13226, 1998.

14. Oh J, Takahashi R, Kondo S, et al: The membrane-anchored MMP inhibitor RECK is a key regulator of extracellular matrix integrity and angiogenesis. Cell 107: 789-800, 2001.

15. Noda M and Takahashi C: Recklessness as a hallmark of aggressive cancer. Cancer Sci 98: 1659-1665, 2007. 
16. Zhang Y, Cheng S, Zhang G, et al: Low expression of RECK indicates a shorter survival for patients with invasive breast cancer. Cancer Sci 103: 1084-1089, 2012.

17. Lin HY, Chiang CH and Hung WC: STAT3 upregulates miR-92a to inhibit RECK expression and to promote invasiveness of lung cancer cells. Br J Cancer 109: 731-738, 2013.

18. Chiang $\mathrm{CH}$, Hou MF and Hung WC: Up-regulation of miR-182 by $\beta$-catenin in breast cancer increases tumorigenicity and invasiveness by targeting the matrix metalloproteinase inhibitor RECK. Biochim Biophys Acta 1830: 3067-3076, 2013.

19. Xin C, Buhe B, Hongting L, et al: MicroRNA-15a promotes neuroblastoma migration by targeting reversion-inducing cysteine-rich protein with Kazal motifs (RECK) and regulating matrix metalloproteinase-9 expression. FEBS J 280: 855-866, 2013.

20. Gabriely G, Wurdinger T, Kesari S, Esau CC, Burchard J, Linsley PS and Krichevsky AM: MicroRNA 21 promotes glioma invasion by targeting matrix metalloproteinase regulators. Mol Cell Biol 28: 5369-5380, 2008.

21. Li N, Tang B, Zhu ED, et al: Increased miR-222 in H. pyloriassociated gastric cancer correlated with tumor progression by promoting cancer cell proliferation and targeting RECK. FEBS Lett 586: 722-728, 2012.

22. Chen C, Ridzon DA, Broomer AJ, et al: Real-time quantification of microRNAs by stem-loop RT-PCR. Nucleic Acids Res 33: e179, 2005
23. Livak KJ and Schmittgen TD: Analysis of relative gene expression data using real-time quantitative PCR and the 2(-Delta Delta C(T)) method. Methods 25: 402-408, 2001.

24. Iorio MV, Casalini P, Tagliabue E, Menard S and Croce CM: MicroRNA profiling as a tool to understand prognosis, therapy response and resistance in breast cancer. Eur J Cancer 44: 2753-2759, 2008.

25. Luo Q, Li X, Li J, et al: MiR-15a is underexpressed and inhibits the cell cycle by targeting CCNE1 in breast cancer. Int $\mathrm{J}$ Oncol 43: 1212-1218, 2013.

26. Li J, Kong X, Zhang J, Luo Q, Li X and Fang L: MiRNA-26b inhibits proliferation by targeting PTGS2 in breast cancer. Cancer Cell Int 13: 7, 2013.

27. Yu S, Lu Z, Liu C, et al: miRNA-96 suppresses KRAS and functions as a tumor suppressor gene in pancreatic cancer. Cancer Res 70: 6015-6025, 2010.

28. Vishwamitra D, Li Y, Wilson D, et al: MicroRNA 96 is a post-transcriptional suppressor of anaplastic lymphoma kinase expression. Am J Pathol 180: 1772-1780, 2012.

29. Chen RX, Xia YH, Xue TC and Ye SL: Suppression of microRNA-96 expression inhibits the invasion of hepatocellular carcinoma cells. Mol Med Rep 5: 800-804, 2012.

30. Calin GA, Liu CG, Sevignani C, et al: MicroRNA profiling reveals distinct signatures in $\mathrm{B}$ cell chronic lymphocytic leukemias. Proc Natl Acad Sci USA 101: 11755-11760, 2004. 